\title{
DETERMINANTS ASSOCIATED TO TRACES ON OPERATOR BIMODULES
}

\author{
K. DYKEMA*, F. SUKOCHEV ${ }^{\S}$, AND D. ZANIN ${ }^{\S}$
}

\begin{abstract}
Given a $\mathrm{II}_{1}$-factor $\mathcal{M}$ with tracial state $\tau$ and given an $\mathcal{M}$-bimodule $\mathcal{E}(\mathcal{M}, \tau)$ of operators affiliated to $\mathcal{M}$ we show that traces on $\mathcal{E}(\mathcal{M}, \tau)$ (namely, linear functionals that are invariant under unitary conjugation) are in bijective correspondence with rearrangement-invariant linear functionals on the corresponding symmetric function space $E$. We also show that, given a positive trace $\varphi$ on $\mathcal{E}(\mathcal{M}, \tau)$, the $\operatorname{map}_{\operatorname{det}}: \mathcal{E}_{\log }(\mathcal{M}, \tau) \rightarrow$ $[0, \infty) \operatorname{defined}^{\mathrm{by}} \operatorname{det}_{\varphi}(T)=\exp (\varphi(\log |T|))$ when $\log |T| \in \mathcal{E}(\mathcal{M}, \tau)$ and 0 otherwise, is multiplicative on the $*$-algebra $\mathcal{E}_{\log }(\mathcal{M}, \tau)$ that consists of all affiliated operators $T$ such that $\log _{+}(|T|) \in \mathcal{E}(\mathcal{M}, \tau)$. Finally, we show that all multiplicative maps on the invertible elements of $\mathcal{E}_{\log }(\mathcal{M}, \tau)$ arise in this fashion.
\end{abstract}

\section{INTRODUCTION}

Let $\mathcal{M}$ be a von Neumann algebra factor of type $\mathrm{II}_{1}$, with tracial state $\tau$. Assume $\mathcal{M}$ has separable predual. The Fuglede-Kadison determinant [8], is the multiplicative map $\Delta_{\tau}: \mathcal{M} \rightarrow[0, \infty)$ defined by

$$
\Delta_{\tau}(T)=\lim _{\epsilon \rightarrow 0^{+}} \exp (\tau(\log (|T|+\epsilon)) .
$$

In this paper, we prove multiplicativity of analogous determinants corresponding to arbitrary positive traces on arbitrary $\mathcal{M}$-bimodules of affiliated operators.

Choose any normal representation of $\mathcal{M}$ on a Hilbert space and let $\mathcal{S}(\mathcal{M}, \tau)$ be the *-algebra of (possibly unbounded) operators on the Hilbert space affiliated to $\mathcal{M}$. This algebra, often called the Murray-von Neumann algebra of $\mathcal{M}$, is independent of the representation. See, for example, Section 6 of [11] for an exposition of this theory. Let $\operatorname{Proj}(\mathcal{M})$ denote the set of projections (i.e., self-adjoint idempotents) in $\mathcal{M}$. For $A \in \mathcal{S}(\mathcal{M}, \tau)$ and $t \in(0,1), \mu(t, A)$ denotes the generalized singular number of $A$, defined by

$$
\mu(t, A)=\inf \{\|A(1-p)\| \mid p \in \operatorname{Proj}(\mathcal{M}), \tau(p) \leq t\},
$$

where $\|\cdot\|$ is the operator norm. This goes back to Murray and von Neumann; see, for example, Section 2.3 of [14 for some basic theory. We will write simply $\mu(A)$ for the function $t \mapsto \mu(t, A)$, which is nonincreasing and right continuous.

Let $E$ be a complex vector space of measurable functions on $[0,1]$ with the property that if $f$ and $g$ are measurable functions with $f^{*} \leq g^{*}$ and $g \in E$, then $f \in E$, where $f^{*}$ denotes the decreasing rearrangement of $|f|$. Following [14, we will call such a space $E$ a Calkin function space. Note that $f \in E$ implies that the dilation $D_{2} f$ lies in $E$, where $D_{2} f(t)=f(t / 2)$. In particular, every nonzero Calkin function space contains $L_{\infty}[0,1]$. The corresponding $\mathcal{M}$-bimodule $\mathcal{E}(\mathcal{M}, \tau)$ is the set of all $A \in \mathcal{S}(\mathcal{M}, \tau)$ such that $\mu(A) \in E$.

Date: September 23, 2016.

2010 Mathematics Subject Classification. 46L52.

Key words and phrases. Determinant, von Neumann algebra, $\mathrm{II}_{1}$-factor, noncommutative function space.

* Research supported in part by NSF grant DMS-1202660.

$\S$ Research supported by ARC. 
This correspondence, sometimes called the Calkin correspondence in the setting of $(\mathcal{M}, \tau)$, is a bijection from the set of all Calkin function spaces onto the set of all operator $\mathcal{M}$ bimodules, by which we mean subspaces of $\mathcal{S}(\mathcal{M}, \tau)$ that are closed under left and right multiplication by elements of $\mathcal{M}$, and it goes back to Guido and Isola [9]. See Theorem 2.4.4 of [14] for the formulation used here. An equivalent version of this is also described in [4]. Note that if $\mathcal{A} \subseteq \mathcal{M}$ is any unital abelian von Neumann subalgebra that is diffuse (i.e., has no minimal projections), then the $*$-algebra $\mathcal{S}\left(\mathcal{A}, \tau \uparrow_{\mathcal{A}}\right)$ of affiliated operators is naturally embedded in $\mathcal{S}(\mathcal{M}, \tau)$ and, upon identifying $\mathcal{A}$ with $L_{\infty}(0,1)$, the elements of $\mathcal{S}\left(\mathcal{A}, \tau \uparrow_{\mathcal{A}}\right)$ are naturally identified with measurable functions on $(0,1)$. Under these identifications, we have $E=\mathcal{S}\left(\mathcal{A}, \tau \uparrow_{\mathcal{A}}\right) \cap \mathcal{E}(\mathcal{M}, \tau)$.

By a trace on $\mathcal{E}(\mathcal{M}, \tau)$, we mean a linear functional $\varphi$ of $\mathcal{E}(\mathcal{M}, \tau)$ such that $\varphi\left(U A U^{*}\right)=$ $\varphi(A)$ for every $A \in \mathcal{E}(\mathcal{M}, \tau)$ and every unitary $U \in \mathcal{M}$. A functional $\varphi_{0}$ of $E$ is said to be rearrangement-invariant if $\varphi_{0}(f)=\varphi_{0}(g)$ whenver $f, g \in E, f, g \geq 0$ and $f^{*}=g^{*}$.

The difficult half of the following result is essentially proved in [13. The proof of the other half is similar to the proof of Lemma 9.4 of [6].

Theorem 1.1. Let $\mathcal{M}$ be a $I I_{1}$-factor with separable predual. Let $E$ be a Calkin function space and let $\mathcal{E}(\mathcal{M}, \tau)$ be the corresponding $\mathcal{M}$-bimodule. There is a bijection from the set of all traces of $\mathcal{E}(\mathcal{M}, \tau)$ onto the set of all rearrangement-invariant functionals of $E$, whereby a trace $\varphi$ of $\mathcal{E}(\mathcal{M}, \tau)$ is mapped to a functional $\varphi_{0}$ of $E$ satisfying

$$
\varphi_{0}(\mu(A))=\varphi(A) \text { whenever } A \in \mathcal{E}(\mathcal{M}, \tau) \text { and } A \geq 0 .
$$

Proof. Suppose $\varphi_{0}: E \rightarrow \mathbb{C}$ is a rearrangement-invariant linear functional. By the proof of (part of) Theorem 5.2 of [13], there is a trace $\varphi: \mathcal{E}(\mathcal{M}, \tau) \rightarrow \mathbb{C}$ satisfying (2). The statement of that theorem includes additional assumptions about $E$, namely, that it carries a rearrangement-invariant complete norm. However, the proof found in [13] is valid, verbatim, in the more general situation considered here.

Suppose $\varphi: \mathcal{E}(\mathcal{M}, \tau) \rightarrow \mathbb{C}$ is a trace. We will now show that for any $A \in \mathcal{E}(\mathcal{M}, \tau)$ that is positive, $\varphi(A)$ depends only on $\mu(A)$. Indeed, let $A_{1}, A_{2} \in \mathcal{E}(\mathcal{M}, \tau)$ be such that $A_{1}, A_{2} \geq 0$ and $\mu\left(A_{1}\right)=\mu\left(A_{2}\right)$. Set

$$
B_{k}=\sum_{n \geq 0} n 1_{[n, n+1)}\left(A_{k}\right), \quad C_{k}=A_{k}-B_{k}, \quad k=1,2 .
$$

Clearly, positive operators $B_{1}$ and $B_{2}$ have discrete spectrum and $\mu\left(B_{1}\right)=\mu\left(B_{2}\right)$. Since $\mathcal{M}$ is a factor, one can choose a unitary element $U \in \mathcal{M}$ such that $B_{1}=U B_{2} U^{-1}$. Clearly, $\varphi\left(B_{1}\right)=\varphi\left(U B_{2} U^{-1}\right)=\varphi\left(B_{2}\right)$. By Theorem 2.3 in [7], we have $\varphi \uparrow_{\mathcal{M}}=c_{\varphi} \tau \uparrow_{\mathcal{M}}$ for a constant $c_{\varphi}$. For bounded positive operators $C_{1}$ and $C_{2}$, we have $\mu\left(C_{1}\right)=\mu\left(C_{2}\right)$ and also, therefore,

$$
\varphi\left(C_{1}\right)=c_{\varphi} \tau\left(C_{1}\right)=c_{\varphi} \tau\left(C_{2}\right)=\varphi\left(C_{2}\right) .
$$

Thus, we get

$$
\varphi\left(A_{1}\right)=\varphi\left(B_{1}\right)+\varphi\left(C_{1}\right)=\varphi\left(B_{2}\right)+\varphi\left(C_{2}\right)=\varphi\left(A_{2}\right) .
$$

Let $\mathcal{A}$ be any unital, diffuse, abelian von Neumann subalgebra of $\mathcal{M}$. As described above, $E$ is naturally identified with $\mathcal{S}\left(\mathcal{A}, \tau \uparrow_{\mathcal{A}}\right) \cap \mathcal{E}(\mathcal{M}, \tau)$, and restricting $\varphi$ to this subalgebra yields a linear functional $\varphi_{0}$ on $E$, which is rearrangement-invariant and satisfies (2), because of the fact that $\varphi(A)$ depends only on $\mu(A)$ for all $A \geq 0$. Using (2), we see that the functional $\varphi_{0}$ does not depend on $\mathcal{A}$, namely, does not depend on which copy of $E$ we chose in $\mathcal{E}(\mathcal{M}, \tau)$.

Finally, as $\varphi$ is uniquely determined by $\varphi_{0}$ and the condition (2), we see that the map $\varphi \mapsto \varphi_{0}$ is the desired bijection. 
For convenience, we will use also $\varphi$, instead of $\varphi_{0}$, to denote the functional on $E$ corresponding to a trace $\varphi$ on $\mathcal{E}(\mathcal{M}, \tau)$.

For example, taking $E$ to be the function space $L_{1}$ of complex-valued functions on $[0,1]$ that are integrable with respect to Lebesgue measure, the corresponding bimodule is $\mathcal{L}_{1}(\mathcal{M}, \tau)$. Moreover, the functional $f \mapsto \int_{0}^{1} f(t) d t$ on $L_{1}$ corresponds to the usual trace $\tau$ on $\mathcal{L}_{1}(\mathcal{M}, \tau)$. Other examples of traces on bimodules are provided by the Dixmier traces on Marcinkiewicz bimodules, which are of interest in noncommutative geometry. See, for example, 3], 2] and [12]; particularly, consider the treatment of functionals supported at zero, but adapted to the case of a $\mathrm{II}_{1}$-factor $\mathcal{M}$, namely, corresponding to function spaces on $[0,1]$. A specific case (essentially, taken from [3]) is found in Example 3.3.

The Fuglede-Kadison determinant mentioned at the start of this introduction is actually naturally defined on the space, sometimes denoted $\mathcal{L}_{\log }(\mathcal{M}, \tau)$, of all $T \in \mathcal{S}(\mathcal{M}, \tau)$ such that $\log _{+}(|T|) \in \mathcal{L}_{1}(\mathcal{M}, \tau)$, where $\log _{+}(t)=\max (\log (t), 0)$. See [10] for a development of $\Delta_{\tau}$ in this generality, including a proof of multiplicativity.

In the rest of this paper, we will for the most part consider only positive traces $\varphi$, namely, those satisfying

$$
A \geq 0 \quad \Longrightarrow \quad \varphi(A) \geq 0
$$

(the exception being Lemma 2.8). Positive traces correspond, under the rubrik of Theorem 1.1, to positive rearrangement-invariant linear functionals. In the following, we use the function $\log _{-}(t)=-\min (\log (t), 0)$; thus, $\log =\log _{+}-\log _{-}$.

Definition 1.2. Let $\mathcal{M}$ be a $\mathrm{II}_{1}$-factor and consider a positive trace $\varphi$ on an $\mathcal{M}$-bimodule $\mathcal{E}(\mathcal{M}, \tau)$. Let $\mathcal{E}_{\log }(\mathcal{M}, \tau)$ be the set of all $T \in \mathcal{S}(\mathcal{M}, \tau)$ such that $\log _{+}(|T|) \in \mathcal{E}(\mathcal{M}, \tau)$ and for such $T$ let

$$
\operatorname{det}_{\varphi}(T)= \begin{cases}\exp (\varphi(\log (|T|))), & \operatorname{ker} T=\{0\} \text { and } \log _{-}(|T|) \in E \\ 0, & \operatorname{ker} T=\{0\} \text { and } \log _{-}(|T|) \notin E \\ 0, & \operatorname{ker} T \neq\{0\} .\end{cases}
$$

Thus, in the case $E=L_{1}$ and $\varphi=\tau$, we have the Fuglede-Kadison determinant: $\operatorname{det}_{\tau}=\Delta_{\tau}$. The natural domain of this determinant by the above rubric should be written $\mathcal{L}_{1, \log }(\mathcal{M}, \tau)$, but we will write $\mathcal{L}_{\log }(\mathcal{M}, \tau)$ for this, in keeping with earlier convention ( $c f$ [5], 6]).

The main result of this paper is:

Theorem 1.3. For an arbitrary Calkin function space $E$ on $[0,1]$ and arbitrary positive trace $\varphi$ on the corresponding bimodule $\mathcal{E}(\mathcal{M}, \tau)$, the set $\mathcal{E}_{\log }(\mathcal{M}, \tau)$ is a *-subalgebra of $\mathcal{S}(\mathcal{M}, \tau)$ and, if $A, B \in \mathcal{E}_{\log }(\mathcal{M}, \tau)$, then

$$
\operatorname{det}_{\varphi}(A B)=\operatorname{det}_{\varphi}(A) \operatorname{det}_{\varphi}(B) .
$$

The proof, presented in the next section, relies on Fuglede and Kadison's result 8$]$ that $\Delta_{\tau}$ is multiplicative on $\mathcal{M}$ and on the characterization from [4] of sums of $\left.(\mathcal{E}(\mathcal{M}, \tau)), \mathcal{M}\right)$ commutators. Thus, a special case of this proof yields an alternative proof of Haagerup and Schultz's result [10] about the extension of the Fuglede-Kadison determinant to $\mathcal{L}_{\log }(\mathcal{M}, \tau)$.

Remark 1.4. It is immediate that $\operatorname{det}_{\varphi}(1)=1$ and, for $T \in \mathcal{E}_{\log }(\mathcal{M}, \tau)$, $\operatorname{det}_{\varphi}(T)=0$ if and only if $T$ fails to be invertible in $\mathcal{E}_{\log }(\mathcal{M}, \tau)$.

Remark 1.5. In the case that $\varphi=0$, we clearly have, for $T \in \mathcal{E}_{\log }(\mathcal{M}, \tau)$,

$$
\operatorname{det}_{\varphi}(T)=\left\{\begin{array}{l}
1 \text { if } T \text { is invertible in } \mathcal{E}_{\log }(\mathcal{M}, \tau) \\
0 \text { otherwise. }
\end{array}\right.
$$


However, if $\varphi \neq 0$, then $\operatorname{det}_{\varphi}$ is onto $[0, \infty)$.

Remark 1.6. It is not difficult to see, in the case $\varphi=\tau$, that Definition 1.2 agrees with the definition by equation (1), in fact even for all $T \in \mathcal{L}_{\log }(\mathcal{M}, \tau)$. However, the analoguous statement is not true for general traces $\varphi$. In fact, it obviously fails when $\varphi=0$, (see Remark 1.5, above). See Example 3.3 for specific examples of this failure when $\varphi \neq 0$.

We are grateful to Amudhan Krishnaswamy-Usha for asking us a question that led to the next result.

Proposition 1.7. For an arbitrary Calkin function space $E$ on $[0,1]$ and an arbitrary map

$$
m: \mathcal{E}_{\log }(\mathcal{M}, \tau) \rightarrow[0, \infty)
$$

that is multiplicative, order-preserving and nonzero, there exists a positive trace $\varphi$ on $\mathcal{E}(\mathcal{M}, \tau)$ such that $m(X)=\operatorname{det}_{\varphi}(X)$ for every invertible element $X$ in $\mathcal{E}_{\log }(\mathcal{M}, \tau)$.

We will show (in Proposition 3.2) that we cannot hope for $m$ to agree with $\operatorname{det}_{\varphi}$ on all of $\mathcal{E}_{\log }(\mathcal{M}, \tau)$.

The proofs of Theorem 1.3 and Proposition 3.2 are contained in the next two sections.

\section{Proof of Theorem 1.3}

Let us begin by describing some further notation and standard conventions.

- $S(0,1)$ will denote the set of all complex-valued Borel measurable functions on $[0,1]$ and $L_{\infty}$ will denote the set of all essentially bounded elements of $S(0,1)$. As usual, we consider functions that are equal almost everwhere to be the same.

- We will apply the Borel functional calculus to self-adjoint elements $T \in \mathcal{S}(\mathcal{M}, \tau)$, and will also use the standard notation $T_{+}=\max (T, 0)$ and $T_{-}=-\min (T, 0)$.

- For self-adjoint $A \in \mathcal{S}(\mathcal{M}, \tau)$, we consider its eigenvalue function (or spectral scale), defined for $t \in(0,1)$ by

$$
\lambda(t, A)=\inf \left\{s \in \mathbb{R} \mid \tau\left(1_{(s, \infty)}(A)\right) \leq t\right\},
$$

where, in accordance with notation for the Borel functional calculus, $1_{(s, \infty)}(A)$ denotes the spectral projection of $A$ associated to the interval $(s, \infty)$. This also goes back to Murray and von Neumann. We will write simply $\lambda(A)$ for the function $t \mapsto \lambda(t, A)$, which is nonincreasing and right continuous. Note that, if $A \geq 0$, then $\lambda(A)=\mu(A)$. Moreover, when $a \leq b$, with $a \leq \lim _{t \rightarrow 0} \lambda(t, A)$ and $b \geq \lim _{t \rightarrow 1} \lambda(t, A)$, we have

$$
\begin{aligned}
\tau\left(A 1_{[a, b]}(A)\right) & =\int_{c}^{d} \lambda(t, A) d t, \\
\tau\left(1_{[a, b]}(A)\right) & =d-c,
\end{aligned}
$$

where

$$
c=\inf \{s \mid \lambda(s, A) \leq b\}, \quad d=\sup \{s \mid \lambda(s, A) \geq a\} .
$$

For any $T \in \mathcal{S}(\mathcal{M}, \tau)$, since $\mu(T)=\mu(|T|)=\lambda(|T|)$, from (5), we get

$$
\tau\left(1_{[0, \mu(t, T)]}(|T|)\right) \geq 1-t .
$$

- The following inequalities are standard (see, for example, Corollary 2.3.16 of [14]): for all $A, B \in \mathcal{S}(\mathcal{M}, \tau)$, if $s, t>0$ and $s+t<1$, then

$$
\begin{aligned}
\mu(s+t, A+B) & \leq \mu(s, A)+\mu(t, B), \\
\mu(s+t, A B) & \leq \mu(s, A) \mu(t, B) .
\end{aligned}
$$


- If a function $f$ on $(0,1)$ is right-continuous and monotone, then we will let $\tilde{f}$ denote left-continuous version, namely,

$$
\tilde{f}(x)=\lim _{t \rightarrow x^{-}} f(t) .
$$

Lemma 2.1. Let $T, S \in \mathcal{S}(\mathcal{M}, \tau)$ be self-adjoint. Then for every $t \in\left(0, \frac{1}{4}\right)$, we have

$$
\left|\int_{2 t}^{1-2 t}\left(\log \left(\mu\left(u, e^{T} e^{S}\right)\right)-\lambda(u, T)-\lambda(u, S)\right) d u\right| \leq 8 t(\mu(t, T)+\mu(t, S)) .
$$

Proof. Fix $t \in\left(0, \frac{1}{4}\right)$ and, using the continuous functional calculus, set

$$
\begin{aligned}
& T_{0}=\min \left\{T_{+}, \mu(t, T)\right\}-\min \left\{T_{-}, \mu(t, T)\right\}, \\
& S_{0}=\min \left\{S_{+}, \mu(t, S)\right\}-\min \left\{S_{-}, \mu(t, S)\right\} .
\end{aligned}
$$

We have

$$
\begin{gathered}
T-T_{0}=\left(T_{+}-\mu(t, T)\right)_{+}-\left(T_{-}-\mu(t, T)\right)_{+}, \\
\left|T-T_{0}\right|=\left(T_{+}-\mu(t, T)\right)_{+}+\left(T_{-}-\mu(t, T)\right)_{+}=(|T|-\mu(t, T))_{+} .
\end{gathered}
$$

Thus, we have $\left(T-T_{0}\right) 1_{[0, \mu(t, T)]}(|T|)=0$ and, using (6) , we get $\mu\left(t, T-T_{0}\right)=0$; similarly, we have $\mu\left(t, S-S_{0}\right)=0$. Using (8), for every $u \in(2 t, 1)$ we have

$$
\begin{aligned}
\mu\left(u, e^{T} e^{S}\right) & =\mu\left(u, e^{T-T_{0}} \cdot e^{T_{0}} e^{S_{0}} \cdot e^{S-S_{0}}\right) \leq \mu\left(t, e^{T-T_{0}}\right) \mu\left(u-2 t, e^{T_{0}} e^{S_{0}}\right) \mu\left(t, e^{S-S_{0}}\right), \\
\mu\left(u, e^{T_{0}} e^{S_{0}}\right) & =\mu\left(u, e^{T_{0}-T} \cdot e^{T} e^{S} \cdot e^{S_{0}-S}\right) \leq \mu\left(t, e^{T_{0}-T}\right) \mu\left(u-2 t, e^{T} e^{S}\right) \mu\left(t, e^{S_{0}-S}\right),
\end{aligned}
$$

Since $\mu\left(t, e^{T-T_{0}}\right) \leq 1$ and $\mu\left(t, e^{T_{0}-T}\right) \leq 1$ and similarly for $S-S_{0}$, we get

$$
\mu\left(u, e^{T} e^{S}\right) \leq \mu\left(u-2 t, e^{T_{0}} e^{S_{0}}\right), \quad \mu\left(u, e^{T_{0}} e^{S_{0}}\right) \leq \mu\left(u-2 t, e^{T} e^{S}\right) .
$$

Thus, for $u \in(2 t, 1-2 t)$, we have

$$
\mu\left(u+2 t, e^{T_{0}} e^{S_{0}}\right) \leq \mu\left(u, e^{T} e^{S}\right) \leq \mu\left(u-2 t, e^{T_{0}} e^{S_{0}}\right) .
$$

It follows that

$$
\int_{4 t}^{1} \log \left(\mu\left(u, e^{T_{0}} e^{S_{0}}\right)\right) d u \leq \int_{2 t}^{1-2 t} \log \left(\mu\left(u, e^{T} e^{S}\right)\right) d u \leq \int_{0}^{1-4 t} \log \left(\mu\left(u, e^{T_{0}} e^{S_{0}}\right)\right) d u .
$$

Since $-\mu(t, T) \leq T_{0} \leq \mu(t, T)$ and similarly for $S_{0}$, we also have

$$
e^{-\mu(t, T)-\mu(t, S)} \leq \mu\left(e^{T_{0}} e^{S_{0}}\right) \leq e^{\mu(t, T)+\mu(t, S)} .
$$

Thus,

In particular,

$$
\begin{gathered}
\left|\int_{0}^{4 t} \log \left(\mu\left(u, e^{T_{0}} e^{S_{0}}\right)\right) d u\right| \leq 4 t\left\|\log \left(\mu\left(e^{T_{0}} e^{S_{0}}\right)\right)\right\|_{\infty} \leq 4 t(\mu(t, T)+\mu(t, S)), \\
\left|\int_{1-4 t}^{1} \log \left(\mu\left(u, e^{T_{0}} e^{S_{0}}\right)\right) d u\right| \leq 4 t\left\|\log \left(\mu\left(e^{T_{0}} e^{S_{0}}\right)\right)\right\|_{\infty} \leq 4 t(\mu(t, T)+\mu(t, S)) .
\end{gathered}
$$

Using (10), we get

$$
\left|\int_{2 t}^{1-2 t} \log \left(\mu\left(u, e^{T} e^{S}\right)\right) d u-\int_{0}^{1} \log \left(\mu\left(u, e^{T_{0}} e^{S_{0}}\right)\right) d u\right| \leq 4 t(\mu(t, T)+\mu(t, S)) .
$$


Since the Fuglede-Kadison determinant $\Delta_{\tau}$ is multiplicative on $\mathcal{M}$, we have

$$
\begin{aligned}
\int_{0}^{1} \log \left(\mu\left(u, e^{T_{0}} e^{S_{0}}\right)\right) d u & =\log \left(\Delta_{\tau}\left(e^{T_{0}} e^{S_{0}}\right)\right) \\
& =\log \left(\Delta_{\tau}\left(e^{T_{0}}\right)\right)+\log \left(\Delta_{\tau}\left(e^{S_{0}}\right)\right)=\tau\left(T_{0}\right)+\tau\left(S_{0}\right) .
\end{aligned}
$$

But using

$$
\left|\tau\left(T_{0}\right)-\int_{2 t}^{1-2 t} \lambda(u, T) d u\right| \leq 4 t \mu(t, T),
$$

and the same also for $S$, the assertion follows.

In the following, we use the notation (9) for the left-continuous versions of monotone functions. (Though, as elements of $E, \mu(T)$ and the left-continuous version $\tilde{\mu}(T)$ are identified, these functions $\mu(T)$ and similarly $\lambda(T)$ are of interest aside from their membership in $E$, and for correctness at all points of $(0,1)$ we must use their left-continuous versions in the following inequalities and elsewhere below.)

Lemma 2.2. If $S, T \in \mathcal{S}(\mathcal{M}, \tau)$ are self-adjoint, then for all $u \in(0,1)$, we have

$$
-\tilde{\mu}\left(\frac{1-u}{2}, T\right)-\tilde{\mu}\left(\frac{1-u}{2}, S\right) \leq \log \left(\mu\left(u, e^{T} e^{S}\right)\right) \leq \mu\left(\frac{u}{2}, T\right)+\mu\left(\frac{u}{2}, S\right) .
$$

Proof. Using (8), we get

$$
\begin{aligned}
\mu\left(u, e^{T} e^{S}\right) \leq \mu\left(\frac{u}{2}, e^{T}\right) \mu\left(\frac{u}{2}, e^{S}\right) \leq \mu\left(\frac{u}{2}, e^{T_{+}}\right) \mu\left(\frac{u}{2}, e^{S_{+}}\right) & =e^{\mu\left(\frac{u}{2}, T_{+}\right)+\mu\left(\frac{u}{2}, S_{+}\right)} \\
& \leq e^{\mu\left(\frac{u}{2}, T\right)+\mu\left(\frac{u}{2}, S\right)}
\end{aligned}
$$

which yields the right-most inequality in (11). Replacing $S$ with $-T$ and $T$ with $-S$ in (12), we get

$$
\mu\left(u, e^{-S} e^{-T}\right) \leq e^{\mu\left(\frac{u}{2}, T_{-}\right)+\mu\left(\frac{u}{2}, S_{-}\right)}, \quad \tilde{\mu}\left(u, e^{-S} e^{-T}\right) \leq e^{\tilde{\mu}\left(\frac{u}{2}, T_{-}\right)+\tilde{\mu}\left(\frac{u}{2}, S_{-}\right)} .
$$

As is well known and easy to show,

$$
\mu\left(u, e^{T} e^{S}\right)=\frac{1}{\tilde{\mu}\left(1-u, e^{-S} e^{-T}\right)} .
$$

Thus, replacing $u$ with $1-u$ in (13), we get

$$
\mu\left(u, e^{T} e^{S}\right) \geq e^{-\tilde{\mu}\left(\frac{1-u}{2}, T_{-}\right)-\tilde{\mu}\left(\frac{1-u}{2}, S_{-}\right)} \geq e^{-\tilde{\mu}\left(\frac{1-u}{2}, T\right)-\tilde{\mu}\left(\frac{1-u}{2}, S\right)},
$$

which yields the left-most inequality in (11).

The next lemma is a combination of Theorems 3.3.3 and 3.3.4 from [14].

Lemma 2.3. If $S, T \in \mathcal{M}$ are positive, then

$$
\int_{0}^{t} \mu(u, T+S) d u \leq \int_{0}^{t}(\mu(u, T)+\mu(u, S)) d u \leq \int_{0}^{2 t} \mu(u, T+S) d u .
$$

Proof. This follows easily from the fact that, for a positive operator, $T$, we have

$$
\int_{0}^{t} \mu(u, T) d u=\sup \{\tau(p T) \mid p \in \operatorname{Proj}(\mathcal{M}), \tau(p) \leq t\} .
$$


For every function $f \in S(0,1)$ that is bounded on compact subsets of $(0,1)$, define

$$
(\Psi f)(t)= \begin{cases}\frac{1}{t} \int_{t}^{1-t} f(s) d s, & 0<t<\frac{1}{2} \\ 0, & \frac{1}{2} \leq t \leq 1 .\end{cases}
$$

Clearly, $\Psi f$ is continuous on $(0,1]$ and $\Psi$ is linear. Note that $\Psi$ is defined on every function arising as $\mu(A)$ or $\lambda(A)$ for $A \in \mathcal{S}(\mathcal{M}, \tau)$.

Lemma 2.4. Let $S, T \in \mathcal{E}(\mathcal{M}, \tau)$ be positive. Then

$$
\Psi(\mu(T+S)-\mu(T)-\mu(S)) \in E .
$$

Proof. First suppose $S, T \in \mathcal{M}$ are positive. From Lemma 2.3 and the fact that $\tau(T)=$ $\int_{0}^{1} \mu(u, T) d u$, we have

$$
\int_{2 t}^{1} \mu(u, T+S) d u \leq \int_{t}^{1}(\mu(u, T)+\mu(u, S)) d u \leq \int_{t}^{1} \mu(u, T+S) d u .
$$

For arbitrary positive $S, T \in \mathcal{S}(\mathcal{M}, \tau)$, set $T_{n}=\min \{T, n\}$ and $S_{n}=\min \{S, n\}$. Since $\mu\left(T_{n}\right) \uparrow \mu(T), \mu\left(S_{n}\right) \uparrow \mu(S)$ and $\mu\left(T_{n}+S_{n}\right) \uparrow \mu(T+S)$, it follows from the Monotone Convergence Principle that (14) also holds. From (14), we have

$$
\left|\int_{t}^{1}(\mu(u, T+S)-\mu(u, T)-\mu(u, S)) d u\right| \leq \int_{t}^{2 t} \mu(u, T+S) d u \leq t \mu(t, T+S) .
$$

Thus, for $t \in\left(0, \frac{1}{2}\right)$, we have

$$
\begin{aligned}
& \left|\int_{t}^{1-t}(\mu(u, T+S)-\mu(u, T)-\mu(u, S)) d u\right| \\
& \leq\left|\int_{t}^{1}(\mu(u, T+S)-\mu(u, T)-\mu(u, S)) d u\right| \\
& \quad+\left|\int_{1-t}^{1}(\mu(u, T+S)-\mu(u, T)-\mu(u, S)) d u\right| \\
& \quad \leq t \mu(t, T+S)+t \mu(1-t, T+S)+t \mu(1-t, T)+t \mu(1-t, S) \leq 4 t \mu(t, T+S) .
\end{aligned}
$$

This concludes the proof.

Lemma 2.5. Let $T \in \mathcal{S}(\mathcal{M}, \tau)$ be self-adjoint. Then

$$
\Psi\left(\lambda(T)-\mu\left(T_{+}\right)+\mu\left(T_{-}\right)\right) \in L_{\infty} .
$$

Proof. If $T_{+}=0$ or $T_{-}=0$, then $\lambda(T)=\mu\left(T_{+}\right)-\mu\left(T_{-}\right)$. Suppose $T_{+} \neq 0$ and $T_{-} \neq 0$. Let $t_{0}$ be the trace of the support projection of $T_{+}$. We have

$$
\lambda(u, T)= \begin{cases}\mu\left(u, T_{+}\right), & u \in\left(0, t_{0}\right) \\ -\tilde{\mu}\left(1-u, T_{-}\right), & u \in\left[t_{0}, 1\right) .\end{cases}
$$

It follows that, for all sufficiently small $t$, we have

$$
\begin{aligned}
t(\Psi \lambda(T))(t)=\int_{t}^{t_{0}} \lambda(u, T) d u & +\int_{t_{0}}^{1-t} \lambda(u, T) d u \\
=\int_{t}^{t_{0}} \mu\left(u, T_{+}\right) d u-\int_{t_{0}}^{1-t} & \mu\left(1-u, T_{-}\right) d u=\int_{t}^{t_{0}} \mu\left(u, T_{+}\right) d u-\int_{t}^{1-t_{0}} \mu\left(u, T_{-}\right) d u \\
& =\int_{t}^{1}\left(\mu\left(u, T_{+}\right)-\mu\left(u, T_{-}\right)\right) d u=t\left(\Psi\left(\mu\left(T_{+}\right)-\mu\left(T_{-}\right)\right)\right)(t),
\end{aligned}
$$


where the last equality holds because the integrand is zero when $u$ is sufficiently close to 1 . Thus, $\Psi\left(\lambda(T)-\mu\left(T_{+}\right)+\mu\left(T_{-}\right)\right)(t)$ vanishes for all $t$ sufficiently small. Since this function is continuous on $(0,1]$, it is bounded.

Lemma 2.6. Let $S, T \in \mathcal{E}(\mathcal{M}, \tau)$ be self-adjoint. Then

$$
\Psi(\lambda(T)+\lambda(S)-\lambda(T+S)) \in E .
$$

Proof. We have

$$
(T+S)_{+}-(T+S)_{-}=T_{+}-T_{-}+S_{+}-S_{-} .
$$

Therefore,

$$
(T+S)_{+}+T_{-}+S_{-}=(T+S)_{-}+T_{+}+S_{+}
$$

Denote the above quantity by $A$. From Lemma 2.4, we obtain

$$
\begin{aligned}
& \Psi\left(\mu(A)-\mu\left((T+S)_{+}\right)-\mu\left(T_{-}\right)-\mu\left(S_{-}\right)\right) \in E, \\
& \Psi\left(\mu(A)-\mu\left((T+S)_{-}\right)-\mu\left(T_{+}\right)-\mu\left(S_{+}\right)\right) \in E .
\end{aligned}
$$

Subtracting those formulae, we obtain

$$
\Psi\left(\mu\left((T+S)_{+}\right)-\mu\left((T+S)_{-}\right)-\mu\left(T_{+}\right)+\mu\left(T_{-}\right)-\mu\left(S_{+}\right)+\mu\left(S_{-}\right)\right) \in E .
$$

The assertion follows now from Lemma 2.5 as applied to the operators $T, S$ and $T+S$, and the fact that $E$ contains $L_{\infty}$.

In the next result, the notation $[\mathcal{E}(\mathcal{M}, \tau), \mathcal{M}]$ denotes the space spanned by the set of all commutators of the form $[S, T]=S T-T S$, for $S \in \mathcal{M}$ and $T \in \mathcal{E}(\mathcal{M}, \tau)$. It amounts to a reformulation of a special case of Theorem 4.6 of [4].

Theorem 2.7. Let $T \in \mathcal{E}(\mathcal{M}, \tau)$ be self-adjoint. Then $T \in[\mathcal{E}(\mathcal{M}, \tau), \mathcal{M}]$ if and only if $\Psi \lambda(T) \in E$.

Proof. By Theorem 4.6 of [4], $T \in[\mathcal{E}(\mathcal{M}, \tau), \mathcal{M}]$ if and only if the function

$$
r \mapsto \frac{1}{r} \tau\left(1_{[0, \mu(r, T)]}(|T|) T\right)
$$

belongs to $E$. Thus, it will suffice to show that the function

$$
r \mapsto \frac{1}{r} \tau\left(1_{[0, \mu(r, T)]}(|T|) T\right)-\Psi \lambda(T)(r)
$$

belongs to $E$. First suppose $T_{-}=0$. Then, using $\lambda(T)=\mu(T)$ and (4), we have

$$
\tau\left(1_{[0, \mu(r, T)]}(|T|) T\right)=\int_{r^{\prime}}^{1} \mu(t, T) d t
$$

where $r^{\prime}=\inf \{s \mid \mu(s, T) \leq \mu(r, T)\}$. Thus $r^{\prime} \leq r$ and, for $0<r<\frac{1}{2}$,

$$
\left|\tau\left(1_{[0, \mu(r, T)]}(|T|) T\right)-\int_{r}^{1-r} \lambda(t, T) d t\right| \leq\left(r-r^{\prime}\right) \mu(r, T)+r \mu(1-r, T) \leq 2 r \mu(r, T),
$$

which implies that the function (15) belongs to $E$.

If $T_{+}=0$, then we may of course replace $T$ by $-T$ and we are done.

Suppose $T_{+} \neq 0$ and $T_{-} \neq 0$. Letting, $t_{0}=\inf \left\{t \mid \lambda\left(t, T_{+}\right) \geq 0\right\}$, we have $0<t_{0}<1$ and

$$
\lambda(t, T)= \begin{cases}\mu\left(t, T_{+}\right), & 0<t<t_{0} \\ \tilde{\mu}\left(1-t, T_{-}\right), & t_{0} \leq t<1\end{cases}
$$


For $r<t_{0}$, we have

$$
\begin{array}{r}
\tau\left(1_{[0, \mu(r, T)]}(|T|) T\right)=\tau\left(1_{[-\mu(r, T), \mu(r, T)]}(T) T\right)=\tau\left(1_{[0, \mu(r, T)]}\left(T_{+}\right) T_{+}\right)-\tau\left(1_{[0, \mu(r, T)]}\left(T_{-}\right) T_{-}\right) \\
=\int_{r^{\prime}}^{t_{0}} \lambda(t, T) d t+\int_{t_{0}}^{1-r^{\prime \prime}} \lambda(t, T) d t
\end{array}
$$

where

$$
\begin{aligned}
& r^{\prime}=\inf \left\{s \mid \mu\left(s, T_{+}\right) \leq \mu(r, T)\right\} \\
& r^{\prime \prime}=\inf \left\{s \mid \mu\left(s, T_{-}\right) \leq \mu(r, T)\right\} .
\end{aligned}
$$

Since $\mu\left(r, T_{ \pm}\right) \leq \mu(r, T)$, we have $r^{\prime}, r^{\prime \prime} \leq r$. Thus, we have

$$
\begin{aligned}
& \left|\tau\left(1_{[0, \mu(r, T)]}(|T|) T\right)-\int_{r}^{1-r} \lambda(t, T) d t\right|=\left|\int_{r^{\prime}}^{r} \lambda(t, T) d t+\int_{1-r}^{1-r^{\prime \prime}} \lambda(t, T) d t\right| \\
& \leq \int_{r^{\prime}}^{r} \mu\left(t, T_{+}\right) d t+\int_{r^{\prime \prime}}^{r} \mu\left(t, T_{-}\right) d t \leq\left(r-r^{\prime}\right) \mu\left(r^{\prime}, T_{+}\right)+\left(r-r^{\prime \prime}\right) \mu\left(r^{\prime \prime}, T_{-}\right) \leq 2 r \mu(r, T),
\end{aligned}
$$

where for the last inequality we used (16)-(17). This shows that the function (15) belongs to $E$ and, thus, completes the proof.

Lemma 2.8. Let $\varphi: \mathcal{E}(\mathcal{M}, \tau) \rightarrow \mathbb{C}$ be a trace. If $T \in \mathcal{E}(\mathcal{M}, \tau)$ is self-adjoint and is such that $\Psi \lambda(T) \in E$, then $\varphi(T)=0$.

Proof. It follows from Theorem 2.7 that $T \in[\mathcal{E}(\mathcal{M}, \tau), \mathcal{M}]$. Since $\varphi$ is a trace, it follows that $\varphi(T)=0$.

Proof of Theorem 1.3. For $A \in \mathcal{S}(\mathcal{M}, \tau)$, we have that $A \in \mathcal{E}_{\log }(\mathcal{M}, \tau)$ if and only if $\log _{+} \mu(A) \in E$, and this is, in turn, equivalent to $\log (1+\mu(A)) \in E$. Using the basic equalities (77)-(8), we easily see that for $A, B \in \mathcal{E}_{\log }(\mathcal{M}, \tau)$, we have

$$
\begin{gathered}
\log (1+\mu(A+B)) \leq \log \left(1+D_{2} \mu(A)+D_{2} \mu(B)\right) \leq \log \left(\left(1+D_{2} \mu(A)\right)\left(1+D_{2} \mu(B)\right)\right) \\
\log (1+\mu(A B)) \leq \log \left(1+D_{2} \mu(A) D_{2} \mu(B)\right) \leq \log \left(\left(1+D_{2} \mu(A)\right)\left(1+D_{2} \mu(B)\right)\right),
\end{gathered}
$$

where $\left(D_{2} f\right)(t)=f(t / 2)$. But since $\log \left(1+D_{2} \mu(A)\right)+\log \left(1+D_{2} \mu(B)\right) \in E$, these imply that $A+B$ and $A B$ belong to $\mathcal{E}_{\log }(\mathcal{M}, \tau)$. From this, one easily sees that $\mathcal{E}_{\log }(\mathcal{M}, \tau)$ is a *-subalgebra of $\mathcal{S}(\mathcal{M}, \tau)$.

It remains to show that $\operatorname{det}_{\varphi}$ is multiplicative. Letting $A, B \in \mathcal{E}_{\log }(\mathcal{M}, \tau)$, we will show (3). We may, without loss of generality, assume $A, B \geq 0$. Indeed, we have $\mu(A B)=$ $\mu\left(|A|\left|B^{*}\right|\right)$. Thus, if the assertion holds for positive operators, then we will have

$$
\operatorname{det}_{\varphi}(A B)=\operatorname{det}_{\varphi}\left(|A|\left|B^{*}\right|\right)=\operatorname{det}_{\varphi}(|A|) \operatorname{det}_{\varphi}\left(\left|B^{*}\right|\right)=\operatorname{det}_{\varphi}(A) \operatorname{det}_{\varphi}(B) .
$$

Suppose first that $\log (A), \log (B) \in \mathcal{E}(\mathcal{M}, \tau)$. Denote, for brevity, $T=\log (A)$ and $S=$ $\log (B)$. It follows from Lemma 2.2 that $\log (|A B|) \in E$.

Using Lemma 2.1 and replacing $t$ with $\frac{1}{2} t$, for all $t \in\left(0, \frac{1}{2}\right)$, we get

$$
\left|\int_{t}^{1-t}\left(\log \left(\mu\left(u, e^{T} e^{S}\right)\right)-\lambda(u, T)-\lambda(u, S)\right) d u\right| \leq 4 t\left(\mu\left(\frac{t}{2}, T\right)+\mu\left(\frac{t}{2}, S\right)\right) .
$$

In particular, we have

$$
\Psi\left(\log \left(\mu\left(e^{T} e^{S}\right)\right)-\lambda(T)-\lambda(S)\right) \in E .
$$

It follows from Lemma 2.6 that

$$
\Psi\left(\lambda\left(\log \left(\left|e^{T} e^{S}\right|\right)-T-S\right)\right) \in E .
$$


Using Lemma 2.8, we conclude that

$$
\varphi\left(\log \left(\left|e^{T} e^{S}\right|\right)-T-S\right)=0
$$

This implies (3) for our $A, B$.

If $B$ has a nonzero kernel, then so does $A B$ and (31) holds.

Suppose now that ker $B$ is zero but $\log _{-}(B) \notin E$. Then, of course, $\lim _{t \rightarrow 1} \mu(t, B)=0$. If ker $A B \neq\{0\}$, then (3) holds, so suppose ker $A B=\{0\}$. We have, from (86), for all $t \in\left(0, \frac{1}{2}\right)$,

$$
\mu(1-t, A B) \leq \mu(t, A) \mu(1-2 t, B)
$$

and, thus,

$$
\log (\mu(1-t, A B)) \leq \log (\mu(t, A))+\log (\mu(1-2 t, B)) .
$$

So, for sufficiently small $t>0$,

$$
\begin{aligned}
\log _{-} \mu(1-t, A B)+\log _{+} \mu(t, A) \geq-\log \mu(1-t, A B)+\log \mu(t, A) & \\
& \geq-\log \mu(1-2 t, B)=\log _{-} \mu(1-2 t, B) .
\end{aligned}
$$

Since the function $t \mapsto \log _{-} \mu(1-2 t, B)$ is not in $E$, while the function $t \mapsto \log _{+} \mu(t, A)$ does belong to $E$, we conclude that the function $t \mapsto \log _{-} \mu(1-t, A B)$ does not belong to $E$. Therefore, the function $\log _{-}(\mu(A B))$ does not belong to $E$ and both left- and right-hand sides of (3) are zero. This concludes the proof of (3) in the degenerate case.

\section{Proof of Proposition 1.7 And some examples}

Lemma 3.1. Let $m: \mathcal{E}_{\log }(\mathcal{M}, \tau) \rightarrow \mathbb{R}$ be multiplicative and order-preserving. Then for every $T \in \mathcal{E}_{\log }(\mathcal{M}, \tau), m(T)$ depends only on $\mu(T)$.

Proof. We may without loss of generality assume $m$ is not identically zero. Thus, $m(1)=1$. By Theorem 1 of [1], every unitary element is a product of multiplicative commutators of unitaries (in fact, of symmetries) and it follows that $m$ sends the entire unitary group of $\mathcal{M}$ to 1 . Thus, by employing the polar decomposition, we have

$$
\forall T \in \mathcal{E}_{\log }(\mathcal{M}, \tau), \quad m(T)=m(|T|) .
$$

It, therefore, suffices to prove the assertion for positive operators.

Let $0 \leq T, S \in \mathcal{E}_{\log }(\mathcal{M}, \tau)$ be such that $\mu(T)=\mu(S)$. Set

$$
T_{\epsilon}=\sum_{n \in \mathbb{Z}}(1+\epsilon)^{n} 1_{\left((1+\epsilon)^{n},(1+\epsilon)^{n+1}\right)}(T), \quad S_{\epsilon}=\sum_{n \in \mathbb{Z}}(1+\epsilon)^{n} 1_{\left((1+\epsilon)^{n},(1+\epsilon)^{n+1}\right)}(S) .
$$

For a given $n$, positive operators $T_{\epsilon}$ and $S_{\epsilon}$ have discrete spectrum and $\mu\left(T_{\epsilon}\right)=\mu\left(S_{\epsilon}\right)$. Since $\mathcal{M}$ is a factor, one can choose a unitary operator $U_{\epsilon} \in \mathcal{M}$ such that $S_{\epsilon}=U_{\epsilon} T_{\epsilon} U_{\epsilon}^{-1}$. Thus,

$$
m\left(S_{\epsilon}\right)=m\left(U_{\epsilon} T_{\epsilon} U_{\epsilon}^{-1}\right)=m\left(U_{\epsilon}\right) m\left(T_{\epsilon}\right) m\left(U_{\epsilon}\right)^{-1}=m\left(T_{\epsilon}\right) .
$$

Clearly,

$$
S_{\epsilon} \leq S \leq(1+\epsilon) S_{\epsilon}, \quad T_{\epsilon} \leq T \leq(1+\epsilon) T_{\epsilon} .
$$

Since $m$ is order preserving, it follows that

$$
m(S) \leq m(1+\epsilon) m\left(S_{\epsilon}\right)=m(1+\epsilon) m\left(T_{\epsilon}\right) \leq m(1+\epsilon) m(T) .
$$

Since $m$ is order preserving, it follows that $m(1+\epsilon) \searrow 1$ as $\epsilon \searrow 0$. Passing $\epsilon \rightarrow 0$, we obtain $m(S) \leq m(T)$. Similarly, $m(T) \leq m(S)$. Thus, $m(S)=m(T)$ and the proof is complete. 
Proof of Proposition 1.7. Since the map $m$ is multiplicative and not identically zero, we must have $m(1)=1$. By Lemma 3.1, $m(T)$ depends only on $\mu(T)$ for all $T \in \mathcal{E}_{\log }(\mathcal{M}, \tau)$.

Let $\mathcal{A}$ be any unital, diffuse, abelian von Neumann subalgebra of $\mathcal{M}$. As in the proof of Theorem 1.3, $E$ is naturally identified with $\mathcal{S}\left(\mathcal{A}, \tau \uparrow_{\mathcal{A}}\right) \cap \mathcal{E}(\mathcal{M}, \tau)$. Given real-valued $f \in E$, let $T \in \mathcal{S}\left(\mathcal{A}, \tau \uparrow_{\mathcal{A}}\right) \cap \mathcal{E}(\mathcal{M}, \tau)$ be the corresponding self-adjoint operator. Note that $e^{T}$ is an invertible element of $\mathcal{E}_{\log }(\mathcal{M}, \tau)$ and, thus, $m\left(e^{T}\right)>0$. We define

$$
\varphi_{0}(f)=\log m\left(e^{T}\right) .
$$

We will show that $\varphi_{0}$ is $\mathbb{R}$-linear. First, given $f_{1}, f_{2} \in E$ and the corresponding self-adjoint $T_{1}, T_{2} \in \mathcal{S}\left(\mathcal{A}, \tau \uparrow_{\mathcal{A}}\right) \cap \mathcal{E}(\mathcal{M}, \tau)$, since $T_{1}$ and $T_{2}$ commute, we have

$$
\varphi_{0}\left(f_{1}+f_{2}\right)=\log m\left(e^{T_{1}+T_{2}}\right)=\log m\left(e^{T_{1}} e^{T_{2}}\right)=\log \left(m\left(e^{T_{1}}\right) m\left(e^{T_{2}}\right)\right)=\varphi_{0}\left(f_{1}\right)+\varphi_{0}\left(f_{2}\right),
$$

i.e., $\varphi_{0}$ preserves addition. From this, we easily see that $\varphi_{0}(r f)=r \varphi_{0}(f)$ for every rational number $r$ and real-valued $f \in E$. This last fact is, of course, equivalent to

$$
m\left(e^{r T}\right)=m\left(e^{T}\right)^{r}
$$

for every self-adjoint $T \in \mathcal{S}\left(\mathcal{A}, \tau \uparrow_{\mathcal{A}}\right) \cap \mathcal{E}(\mathcal{M}, \tau)$ and every rational number $r$. When $T \geq 0$, using the order-preserving property of $m$, we obtain from this that (19) holds for every $r \in \mathbb{R}$, and similarly when $T \leq 0$. For arbitrary self-adjoint $T \in \mathcal{S}\left(\mathcal{A}, \tau \uparrow_{\mathcal{A}}\right) \cap \mathcal{E}(\mathcal{M}, \tau)$, writing $T=T_{+}-T_{-}$for $T_{+}$and $T_{-}$positive elements of $\mathcal{S}\left(\mathcal{A}, \tau \uparrow_{\mathcal{A}}\right) \cap \mathcal{E}(\mathcal{M}, \tau)$, in the usual way, we get, for all $r \in \mathbb{R}$,

$$
\begin{aligned}
m\left(e^{r T}\right)=m\left(e^{r T_{+}+(-r) T_{-}}\right)=m\left(e^{r T_{+}}\right) m\left(e^{(-r) T_{-}}\right)=m\left(e^{T_{+}}\right)^{r} m\left(e^{T_{-}}\right)^{-r} & \\
& =\left(m\left(e^{T_{+}} e^{-T_{-}}\right)\right)^{r}=m\left(e^{T}\right)^{r} .
\end{aligned}
$$

Thus (19) holds for all self-adjoint $T$ and all $r \in \mathbb{R}$, and it follows that $\varphi_{0}(r f)=r \varphi_{0}(f)$ for all real-valued $f \in E$ and all $r \in \mathbb{R}$. Thus, we have defined an $\mathbb{R}$-linear functional $\varphi_{0}$ on the space of real-valued elements of $E$. Complexification extends $\varphi_{0}$ to a $\mathbb{C}$-linear functional on $E$.

We now observe that $\varphi_{0}$ is rearrangement-invariant. If $f \in E$ and $f \geq 0$ and if $T \in$ $\mathcal{S}\left(\mathcal{A}, \tau \uparrow_{\mathcal{A}}\right) \cap \mathcal{E}(\mathcal{M}, \tau)$ is the corresponding element, then $\mu\left(e^{T}\right)=e^{f^{*}}$, where $f^{*}$ is the nondecreasing rearrangement of $f$. Since $m\left(e^{T}\right)$ depends only on $\mu\left(e^{T}\right)$, we see that $\varphi_{0}(f)=$ $\varphi_{0}\left(f^{*}\right)$ and, thus, $\varphi_{0}$ is rearrangement-invariant.

By Theorem 1.1, there is a unique trace $\varphi$ on $\mathcal{E}(\mathcal{M}, \tau)$ such that $\varphi(T)=\varphi_{0}(\mu(T))$ whenever $T \in \mathcal{E}(\mathcal{M}, \tau)$ is positive. Suppose $X$ is an invertible element of $\mathcal{E}_{\log }(\mathcal{M}, \tau)$ and let us observe that $m(X)=\operatorname{det}_{\varphi}(X)$. Since $m(X)=m(|X|)$ and likewise for $\operatorname{det}_{\varphi}$, we may without loss of generality assume $X \geq 0$. Thus, there is self-adjoint $T=\log (X) \in \mathcal{E}(\mathcal{M}, \tau)$ such that $X=e^{T}$. Thus, by (18), we have

$$
m(X)=e^{\varphi_{0}(\lambda(T))}=e^{\varphi(T)}=\operatorname{det}_{\varphi}(X),
$$

as required.

The following shows that Proposition 1.7 cannot be improved to obtain $m=\operatorname{det}_{\varphi}$ on all of $\mathcal{E}_{\log }(\mathcal{M}, \tau)$.

Proposition 3.2. Let $E$ be a symmetric function space. Consider strictly larger symmetric function space $F$. If $\psi$ is an arbitrary positive trace on $\mathcal{F}(\mathcal{M}, \tau)$, then

$$
\operatorname{det}_{\psi} \Upsilon_{\mathcal{E}_{\log }(\mathcal{M}, \tau)} \neq \operatorname{det}_{\varphi}
$$

for each positive trace $\varphi$ on $\mathcal{E}(\mathcal{M}, \tau)$. 
Proof. To see this, fix $0 \leq T \in \mathcal{F}(\mathcal{M}, \tau)$ such that $T \notin \mathcal{E}(\mathcal{M}, \tau)$. Take $X=e^{-T}$. Then $X$ is bounded, so belongs to $\mathcal{E}_{\log }(\mathcal{M}, \tau)$. Moreover, $X^{-1}=e^{T}$ belongs to $\mathcal{F}_{\log }(\mathcal{M}, \tau)$, but $X$ is not invertible in $\mathcal{E}_{\log }(\mathcal{M}, \tau)$. Thus, we have

$$
\operatorname{det}_{\psi}(X)=e^{-\psi(T)} \neq 0=\operatorname{det}_{\varphi}(X) .
$$

See Remark 1.6 for the relevance of the following example.

Example 3.3. We give examples of a nonzero trace $\varphi$ on a bimodule $\mathcal{E}(\mathcal{M}, \tau)$ and $T \in$ $\mathcal{E}(\mathcal{M}, \tau)$ such that $\varphi \neq 0$ but

$$
\operatorname{det}_{\varphi}(T) \neq \lim _{\epsilon \rightarrow 0^{+}} \operatorname{det}_{\varphi}(|T|+\epsilon) .
$$

Let $\psi$ be an increasing, continuous, concave function on the interval $[0,1]$ satisfying

$$
\lim _{t \rightarrow 0} \frac{\psi(2 t)}{\psi(t)}=1 .
$$

For example, take $\psi(t)=\frac{1}{2-\log (t)}$. Let $E=M_{\psi}$ be the Marcinkiewicz space

$$
E=\left\{\begin{array}{l|l}
f \in S(0,1) & \left.\sup _{0<t<1} \frac{1}{\psi(t)} \int_{0}^{t} f^{*}(s) d s<\infty\right\}
\end{array}\right.
$$

where $f^{*}$ is the decreasing rearrangement of $|f|$. Let $\mathcal{E}(\mathcal{M}, \tau)$ be the corresponding $\mathcal{M}$ bimodule. By Example 2.5(ii) of [3, there is a positive, rearrangement-invariant, linear functional $\varphi$ on $E$ that vanishes on $E \cap L_{\infty}$, but satisfies $\varphi\left(\psi^{\prime}\right)=1$. For $f \in E$ with $f \geq 0$, $\varphi(f)$ is realized as a particular sort of generalized limit as $t \rightarrow 0$ of $\frac{1}{\psi(t)} \int_{0}^{t} f^{*}(s) d s$. Let $\varphi$ denote also the trace on $\mathcal{E}(\mathcal{M}, \tau)$, according to Theorem 1.1. Thus, we have $\operatorname{det}_{\varphi}(T)=1$ whenever $T \in \mathcal{M}$ is bounded and has bounded inverse. Consequently, if $T \in \mathcal{M}$ fails to be invertible in $\mathcal{E}_{\log }(\mathcal{M}, \tau)$, for example, because it has a nonzero kernel, then, by Definition 1.2. $\operatorname{det}_{\varphi}(T)=0$, but the right-hand-side of (20) is equal to 1 .

The examples considered hitherto involved non-invertible elements of $\mathcal{E}_{\log }(\mathcal{M}, \tau)$. However, (20) can also fail when $T$ is invertible in $\mathcal{E}_{\log }(\mathcal{M}, \tau)$. For example, take $T \geq 0$ such that $\mu(T)(t)=\exp \left(-\psi^{\prime}(1-t)\right)$. In particular, $T$ is bounded. Then $\operatorname{det}_{\varphi}(T)=e^{-1}$ but again the right-hand-side of (20) is equal to 1.

Acknowldegement: The authors thank Amudhan Krishnaswamy-Usha for valuable discussions.

\section{REFERENCES}

[1] M. Broise, Commutateurs dans le groupe unitaire d'un facteur, J. Math. Pures Appl. (9) 46 (1967), 299-312.

[2] A. L. Carey and F. A. Sukochev, Dixmier traces and some applications to noncommutative geometry, Uspekhi Mat. Nauk 61 (2006), no. 6(372), 45-110 (Russian, with Russian summary); English transl., Russian Math. Surveys 61 (2006), 1039-1099.

[3] P. G. Dodds, B. de Pagter, E. M. Semenov, and F. A. Sukochev, Symmetric functionals and singular traces, Positivity 2 (1998), 47-75.

[4] K. J. Dykema and N. J. Kalton, Sums of commutators in ideals and modules of type II factors, Ann. Inst. Fourier (Grenoble) $\mathbf{5 5}$ (2005), 931-971.

[5] K. Dykema, F. Sukochev, and D. Zanin, Algebras of log-integrable functions and operators, Complex Anal. Oper. Theory, to appear, available at arXiv:1509.03360

[6] _ An upper triangular decomposition theorem for some unbounded operators affiliated to $I_{1}$ factors, available at arXiv:1509.03358. 
[7] T. Fack and P. de la Harpe, Sommes de commutateurs dans les algèbres de von Neumann finies continues, Ann. Inst. Fourier (Grenoble) 30 (1980), 49-73.

[8] B. Fuglede and R. V. Kadison, Determinant theory in finite factors, Ann. of Math. 55 (1952), 520-530.

[9] D. Guido and T. Isola, Singular traces on semifinite von Neumann algebras, J. Funct. Anal. 134 (1995), 451-485.

[10] U. Haagerup and H. Schultz, Brown measures of unbounded operators affiliated with a finite von Neumann algebra, Math. Scand. 100 (2007), 209-263.

[11] R. V. Kadison and Z. Liu, The Heisenberg relation-mathematical formulations, SIGMA Symmetry Integrability Geom. Methods Appl. 10 (2014), Paper 009, 40.

[12] N. J. Kalton, A. A. Sedaev, and F. A. Sukochev, Fully symmetric functionals on a Marcinkiewicz space are Dixmier traces, Adv. Math. 226 (2011), 3540-3549.

[13] N. J. Kalton and F. A. Sukochev, Rearrangement-invariant functionals with applications to traces on symmetrically normed ideals, Canad. Math. Bull. 51 (2008), no. 1, 67-80.

[14] S. Lord, F. Sukochev, and D. Zanin, Singular Traces, de Gruyter Studies in Mathematics, vol. 46, Walter de Gruyter \& Co., Berlin, 2013.

K. Dykema, Department of Mathematics, Texas A\&M University, College Station, TX, USA.

E-mail address: ken.dykema@math.tamu.edu

F. Sukochev, School of Mathematics and Statistics, University of New South Wales, Kensington, NSW, Australia.

E-mail address: f.sukochev@math.unsw.edu.au

D. Zanin, School of Mathematics and Statistics, University of New South Wales, KensingTON, NSW, Australia.

E-mail address: d.zanin@math.unsw.edu.au 